\title{
Structural and Functional Characteristics of Soil Microbial Community in a Pinus Massoniana Forest at Different Elevations
}

Jian Zhang ( $\square$ zhangjian12102@163.com )

Guizhou University

Ming Xu

Guizhou University

Xiao Zou

Guizhou University

\section{Research}

Keywords: Subtropical Forest, Pinus massoniana, Soil Microbial Communities, Metagenome, Structure and function, Elevational gradients

Posted Date: October 15th, 2020

DOI: https://doi.org/10.21203/rs.3.rs-91192/v1

License: (c) (1) This work is licensed under a Creative Commons Attribution 4.0 International License. Read Full License 


\section{Abstract}

\section{Background}

In spite of the ecological importance of soil microbial communities, very little data is available on different soil microorganism types along altitudinal gradients on a mountain system with a forest ecosystem to identify the distribution patterns of soil microbial diversity and function. This study aimed to determine the diversity patterns of different soil microorganism types (archaea, bacteria, fungi, viruses) along an altitudinal gradient on Mt. Leigong, a typical mid-subtropical mountain forest ecosystem.

Results

The richness of bacterial, fungal, and virus community changed in a unimodal pattern, while archaeal community changed in a bimodal pattern with increasing altitude. Euryarchaeota and Thaumarchaeota, Proteobacteria and Acidobacteria, Ascomycota and Basidiomycota, Myoviridae and Podoviridae were abundant taxa in archaeal, bacterial, fungal, and virus community, respectively. Amino acid transport and metabolism, and energy production and conversion were the predominant categories as per Nonsupervised Orthologous Groups (NOG) function gene-annotation. Carbohydrate metabolism, global and overview map, and amino acid metabolism, were predominant categories in the Kyoto encyclopedia of genes and genomes-based (KEGG) pathways. Glycosyl transferase and glycoside hydrolase were predominant categories among carbohydrate enzyme-functional genes. Cluster analysis, redundancy analysis, and Network analysis showed obvious differences in composition, structure, and function of different soil microorganism types in the P. massoniana forest on Mt. Leigong.

\section{Conclusions}

These results indicate that different soil microorganism types (archaea, bacteria, fungi, viruses) along the altitudinal gradient have an obvious different distribution patterns in the typical mid-subtropical mountain coniferous forest soil.

\section{Introduction}

Natural vertical gradients associated with altitude in mountain ecosystems have attracted great interest from ecologists around the world because these habitats show characteristics of altitudinal gradients, whereby rapid changes in climate and biological characteristics within a short geographic range are commonly observed (Siles \& Margesin 2016). For example, if ambient temperature decreases with increasing altitude, precipitation, solar radiation, and atmospheric pollution deposits change due to the changing elevation of the terrain, whereby complex ecological changes occur along the altitude gradient (Körner 2007). Therefore, the mechanism underlying changes in species richness along an elevation gradient has always been a controversial issue in ecological and biogeographic studies. Although there are reports of diminishing patterns or single-peak patterns for animal and plant mechanisms responsive to elevation, such differences may show stronger variation among different regions in the case 
microorganisms, including no trend, a decline, a single peak, or a concave or other distribution models associated with changes in altitude (Siles \& Margesin 2016; Liu et al. 2018). In recent years, research on altitudinal patterns of microbial diversity (i.e., bacteria, fungi, ectomycorrhizal fungi, etc.) has gradually increased by using high-throughput sequencing technology (Jarvis et al. 2015; Wang et al. 2015; Geml et al. 2017; Liu et al. 2018; Schoen et al. 2018).

Soil microorganisms are important biological components associated with aboveground interactions of terrestrial ecosystems (Bahram et al. 2018), with microorganisms being key drivers of litter decomposition, plant growth, soil nutrient cycling and biogeochemical process (van der Heijden \& Wagg 2013; Bardgett \& van der Putten 2014; Schloter et al. 2018; Thakur \& Geisen 2019). Due to the importance of forest soils as both sinks and potential sources of carbon and their predicted sensitivity to climate change, microbial ecologists have been struggling to indicate that what is the interaction mechanism between environment factors (topography, climate, vegetation and soil) and soil microbial communities (diversity and function) in forest ecosystem (Lladó et al. 2018). Topography, as a long-term constant factor, have a significant impact on ecosystem dynamics, which may lead to changes in soil microbial communities (Qiu et al. 2012; Zhang et al. 2013; Tajik et al. 2020). From a biological perspective, topographic parameters can provide accurate and valuable information that is useful for predicting the distribution of biodiversity of soil microbial communities (Lladó et al. 2018; Tajik et al. 2020). Therefore, promoting the study of the mechanism of distribution of soil microorganisms along altitude gradients in mountain ecosystems should prove useful for a scientifically based management practice of mountain ecosystems.

Mountainous landforms are very common in Southwest China, which increases habitat heterogeneity while providing an important foundation for biodiversity richness (Myers et al. 2000). Pinus massoniana is an important native tree species and the most widely distributed pine tree in Southern China, with strong adaptability and tolerance to harsh environments (Lei et al. 2018). P. massoniana forest is the main vegetation type with key ecological and economic importance (such as vegetation construction, timber, resin, etc.) in Southern China. Masson pine forests are distributed along elevation gradients which provide natural experimental locations for trying to elucidate the elevation-associated mechanism of soil microbial-communities distribution. In this paper, soil microorganisms of the $P$. massoniana forest at different altitudes in Leigong Mountain, Guizhou Province, China, were selected as the research object. Species diversity composition and function of soil microbial communities were analyzed by Illumina Meseq metagenome sequencing technology in order to provide a scientific basis for the ecological management and utilization of Masson pine forests in the region.

\section{Materials And Methods}

\subsection{Site Description}

Leigong Mountain Nature Reserve $\left(26^{\circ} 20^{\prime} 25^{\prime \prime}-26^{\circ} 25^{\prime} 00^{\prime \prime} \mathrm{N}\right.$; $108^{\circ} 12^{\prime} 00^{\prime \prime}$ - $108^{\circ} 20^{\prime} 00^{\prime \prime}$ E) was established as a provincial Nature Reserve in 1982, and was promoted to National Nature Reserve in 2001. Leigong 
Mountain (2178.8 $\mathrm{m}$ a.s.I.) is the main peak among the Miaoling Mountains, which constitute the drainage divide between the Pearl River basin and the Yangtze River basin. With 83\% forest coverage, this mountain range is an ideal area to study the typical mid-subtropical mountain forest ecosystem (Zhang et al. 2020). Leigong Mountain belongs to the humid climatic region of the mid-subtropical monsoon mountainous region, with a characteristic warm and humid climate with abundant rainfall, many rain and fog days, and low light levels. The annual rainfall is $1300 \sim 1600 \mathrm{~mm}$; the annual average temperature is about 9.2 and $16.3^{\circ} \mathrm{C}$ at the top and at the foot of the mountain, respectively, with an annual average temperature drop rate of $0.46{ }^{\circ} \mathrm{C}$ per $100 \mathrm{~m}$. The soils are mainly acidic mountain yellow soils and mountain yellow brown soils, with a deep soil profile. The boundary between these two soil types lies at approximately $1300 \mathrm{~m}$ a.s.I. The zonal vegetation belongs to the humid evergreen broad-leaved forest in the eastern mid-subtropical zone, where it is distributed below $1350 \mathrm{~m}$, while the mountain evergreen deciduous broad-leaved mixed forest is distributed between 1350 and $2100 \mathrm{~m}$ a.s.l., above which the alpine shrub is observed.

\subsection{Study Site and Soil Sampling}

In June 2019, four similar points were selected along an altitudinal gradient across the P. massoniana forest in Leigong Mountain: namely, 1000, 1200, 1400, and 1600 m a.s.I.-Mp1000, Mp1200, Mp1400, and Mp1600, respectively. An artificial tea garden (Tea-garden) was selected as control at $1000 \mathrm{~m}$ a.s.l. One vegetation survey plot with $20 \mathrm{~m} \times 20 \mathrm{~m}$ in size were set at each altitude. Our survey included information on site conditions, plant communities, and litter in the sample plot. Soil samples were excavated from the 0-20 cm topsoil layer at six points located along an "S" path drawn within each plot, and thoroughly mixed. Detailed information about these samples is listed in Table 1. For each sample, $500 \mathrm{~g}$ of soil was sieved though a 2-mm mesh and stored at $4{ }^{\circ} \mathrm{C}$ for physicochemical analyses; additionally, $50 \mathrm{~g}$ of soil was encapsulated in a plastic tube and kept at $-80^{\circ} \mathrm{C}$ before microbial DNA extraction.

\subsection{Soil Analysis and Microbial DNA Extraction}

Soil $\mathrm{pH}$ was determined at a ratio of 1:2.5 (soil to water, w/w). Soil organic carbon (SOC) was determined using the $\mathrm{K}_{2} \mathrm{Cr}_{2} \mathrm{O}_{7}$ titration method (Walkley-Black method). Total nitrogen (TN) was measured using the semi-micro Kjeldahl method. Total phosphorus (TP) was determined colorimetrically after wet digestion with $\mathrm{H}_{2} \mathrm{SO}_{4}+\mathrm{HClO}_{4}(\mathrm{Xu}$, et al., 2014).

Total genomic DNA was isolated from $0.50 \mathrm{~g}$ of soil using the MoBio Ultraclean Soil DNA Isolation Kit (MoBio laboratories, Carlsbad, California, USA) following the instructions of the manufacturer. Extracted DNA was stored at $-80^{\circ} \mathrm{C}$, and tenfold diluted DNA samples were used for metagenome analyses on an Illumina MiSeq platform (Illumina, San Diego, USA) according to the standard protocols of Majorbio BioPharm Technology Co. Ltd. (Shanghai, China).

\subsection{Metagenome Sequencing}


The Illumina sequencing platform was used to perform parallel mixing and sequencing of multiple samples. The sequence in each sample was introduced with an Index tag sequence indicating sample source information. Software "Seqprep" was used to cut the 3 ' end and 5 ' the adapter sequence at the end. Software "Sickle" was used to remove the cut length less than $50 \mathrm{bp}$; the average quality value was below a certain threshold (default 20) and N-base reads; high-quality pair-end reads and single-end reads were retained (Pabinger et al. 2014; chirmer, et al. 2015). We then used fastp (https://github.com/OpenGene/fastp) software to perform quality control processing on the original sequencing data to obtain high-quality control data (clean data), and Megahit (https://github.com/voutcn/ megahit) software to assemble sequences with different sequencing depths (Li et al. 2015), and to preserve sequences with assembly contigs $>300 \mathrm{bp}$. MetaGene (http://metagene.cb.ku-tokyo.ac.jp/) was then used to predict Open Reading Frames (ORF) of contigs $\geq$ $100 \mathrm{bp}$ in the stitched result, and CD-HIT software (http:/ /www.bioinformatics.org/cd-hit/) for clustering (identity $\geq 90 \%$ and coverage $\geq 90 \%$ ), taking the longest gene in each class as a representative sequence to construct a non-redundant gene set (Noguchi et al. 2006). SOAPaligner software

(http://soap.genomics.org.cn/) was used to compare the high-quality reads of each sample with the nonredundant gene set (identity $\geq 95 \%$ ), and to count gene abundance information in the corresponding samples (Luo et al. 2012). In turn, BLASTP software (http://blast.ncbi.nlm.nih.gov/Blast.cgi) was used to separate non-redundant gene sets with NR database (Non-Redundant Protein Sequence Database) (Altschul et al. 1997)and eggNOG database (Evolutionary genealogy of genes: Nonsupervised Orthologous Groups, http://eggnog.embl.de/) (Powell et al. 2012), KEGG's Gene Database (GENES) and CAZy (Carbohydrate-active enzymes, http://www.cazy.org/, Version 5.0) Annotate species and functions, and set the expected e-value to 1e-5 (Du et al. 2014; Lombard et al. 2014).

Subsequently, R language was used to count the total and unique species, functions or genes of archaea, bacteria, fungi, and virus groups in different sample-soil microbial communities, and to draw a Wayne diagram; count the archaea, bacteria, fungi, and viruses in the soil microbial community. The horizontal community histogram was based on cluster analysis of the similarity between the samples and the soil microbial archaea, bacteria, fungi, and virus groups. Similarity results of the soil microbial community among different soil samples are presented by a heatmap. Test analyses were performed to test the correlation between the soil microbial community matrix and the function matrix among different samples. The soil microbial community species-level was based on the Bray-Curtis distance algorithm for RDA analysis. The networks software was used for co-occurrence network analysis (Barberan et al. 2012). Soil environmental factors were analyzed using SPSS 22.5 One-way ANOVA statistics. Finally, we used Sigma Plot 12.5 and Adobe Illustrator software for drawing processing details.

\section{Results}

\subsection{Composition and Structure of Soil Microbial Communities}

A total of 452526750 original sequences were obtained from the metagenomic sequencing of soil microorganisms in the $P$. massoniana forest along the altitudinal gradient established. After quality 
control, a total of 447074316 effective sequences were generated, and the sub-effective sequences were selected for different soil microbial groups, including archaea, bacteria, fungi, and viruses. There were 62 208980 effective sequences and a single sample produced 9960 236 16 161802 effective sequences. After metagenomic sequencing and NR (non-redundant protein sequences) database species-annotation, a total of 13307 soil microorganisms were obtained. These included 912 archaea, 11576 bacteria, 417 fungi, and 402 virus species (Table 2).

The changes in richness and Shannon index of soil microbial groups at the different altitudes selected along the altitudinal gradient marked across the $P$. massoniana forest at the study site showed different trends (Table 3). Specifically, in terms of species richness, the abundance of soil bacteria, fungi, and viruses exhibited a "unimodal" pattern with altitude, while archaea showed a significant peak at Mp1200 (752) and a secondary peak at Mp1600 (716). The Shannon index for archaea and fungi increased with increasing altitude, whereas the Shannon index for bacteria decreased with increasing altitude. The Shanon index for viruses did not show any obvious pattern of change with altitude.

The distribution of common species and endemic species of different soil microbial groups in different sites showed significantly different profiles (Fig. 1). Common species of archaea, bacteria, fungi, and viruses accounted for $58.6 \%, 75.1 \%, 50.4 \%$, and $7.5 \%$ of the soil microbial groups, respectively. Whereas endemic species of archaea, bacteria, fungi, and viruses accounted for $12.3 \%, 6.6 \%, 15.6 \%$, and $54.5 \%$, respectively. These results indicated that the spatial distribution pattern of different soil microbial groups across altitudes differed significantly. Thus, for example, archaea and fungi showed similar patterns in their distribution but there were obvious differences between those of bacteria and viruses.

Euryarchaeota and Thaumarchaeota were the predominant groups of soil archaea in the P. massoniana forest across the altitudinal gradient surveyed. The order of relative abundance of these two groups at the five sites was: Tea-garden (84.1\%) > Mp1000 (80.3\%) > Mp1200 (75.8\%) > Mp1400 (75.6\%) > Mp1600 (75.5\%). Euryarchaeota and Thaumarchaeota were followed in relative abundance by Candidatus Bathyarchaeota, Crenarchaeota, Candidatus Thorarchaeota, Candidatus Lokiarchaeota, Candidatus Micrarchaeota, Candidatus Korarchaeota, Candidatus Parvarchaeota, Candidatus Nanohaloarchaeota and Nanoarchaeota (Fig. 2a).

Proteobacteria and Acidobacteria were the predominant groups of bacteria in the soils of the $P$. massoniana forest across the altitudinal gradient established in this study. The order of relative abundance of these two bacterial groups at the five sites was: Mp1000 $(73.1 \%)<$ Tea-garden $(74.0 \%)<$ Mp1200 (74.3\%) < Mp1600 (76.0\%) < Mp1400 (76.2\%), with a slightly increasing trend with increasing altitude. The bacterial groups following in relative abundance were Actinobacteria, Verrucomicrobia, Chloroflexi, Planctomycetes, Cyanobacteria, Firmicutes, Bacteroidetes, Gemmatimonadetes and other bacteria (Fig. 2b).

Ascomycota and Basidiomycota were the predominant groups in the P. massoniana forest across the altitudinal gradient. The order of relative abundance of these two fungal groups at the five sites was: Teagarden $(89.4 \%)<(92.2 \%)<$ Mp1200 (93.3\%) < Mp1400 (94.2\%) < Mp1600 (94.4\%), with a slightly 
increasing trend with increasing altitude. Thus, ascomycetes increased with elevation and their relative abundance decreased significantly; while basidiomycete showed an obvious increase with elevation; and the relative abundance of the two phylum groups has a stable upward trend with increasing altitude. The fungal groups following in relative abundance were Chytridiomycota, Glomeromycota, Blastocladimycota, Entomophthoromycota, Cryptomycota, Microsporidia, Neocallimastigomycota and other fungi (Fig. 2c).

Myoviridae, Podoviridae, and Siphoviridae were the predominant virus groups in the $P$. massoniana forest across the altitudinal gradient established for this survey. The order of relative abundance of these viral groups at the five sites was: Mp1600 (42.1\%) < Tea-garden (48.7\%) < Mp1000 (49.6\%) < Mp1200 (50.8\%) $<$ Mp1400 (54.5\%). The groups following in relative abundance were Phycodnaviridae, Tectiviridae, Mimiviridae, unclassified_d_ virus, Caudovirales and other viruses (Fig. 2d).

Clustering of different soil microbial groups (archaea, bacteria, fungi and viruses) at genus level showed significant differences and most genera present in different soil microbial groups changed highly significantly with altitude. Overall, Candidatus_Nitrosotalea, unclassified_p_Candidatus_Bathyarchaeota, Methanosarcina, Nitrososphaera, and unclassified_d_Archaea were the predominant archaeal genera (Fig. 3a), while unclassified_f_Acidobacteriaceae, Candidatus_Solibacter, Candidatus_Koribacter and Bradyrhizobium were the predominant bacterial genera (Fig. 3b). In turn, Moniliophthora, Penicillium, Oidiodendron, Colletotrichum, Aspergillus and Cladophialophora were the predominant fungal genera (Fig. 3c). Unclassified_d_Viruses, unclassified_f_Myoviridae, unclassified_f_Siphoviridae and unclassified_f_Podoviridae were the predominant virus genera (Fig. 3d). Archaea and fungal genera present in soil microbial communities showed a relatively more similar trend of change along altitude.

\subsection{Functional Gene Composition and Structure of Soil Microbial Communities}

The BLASTP function prediction based on eggNOG database was used to determine the soil microbial community functions in a $P$. massoniana forest at different altitudes. The main groups of identified genes corresponded to those with functions involved in amino acid transport and metabolism (7.51-7.98\%), energy production and transformation (6.62-7.46\%), signal transduction mechanisms (6.76-7.24\%), and DNA replication, recombination, and repair (6.46-7.16\%). These were the dominant gene categories and, clearly, these functions are important for energy production and cell biology. In addition, a group of genes with unknown functions accounted for $30 \%$ (28.58-31.16\%) of all genes classified (Fig. 4).

A quantitatively high representation of carbohydrate metabolism (12.2-13.0\%), global and overview map (11.5-11.6\%), amino acid metabolism (10.2-10.7\%), and energy metabolism (7.5-7.8\%) was observed when the KEGG database was applied to the classification of the identified genes into putative pathways (Fig. 5a). Glycosyl transferase (GT, 34.59-38.58\%) and glycoside hydrolase (GH, 28.98\%-33.59\%) were the highest proportions in carbohydrate active enzymes (CAZy), followed by carbohydrate esterase (CE, 15.91-16.28\%) and coenzyme activity (AA, 10.43-10.92\%), and polysaccharide lyase (PL, 3.00-3.64\%), combined with the carbohydrate Module (CBM, 1.98-2.14\%)(Fig. 5b). 


\subsection{Similarity of Species and Functions Among Soil Microbial Communities}

The results of similarity and dissimilarity of species as per NR, NOG, KO and CAZy functional genes among all sample sites were compared to results obtained from similarity clustering analysis and redundancy analysis (RDA). Figure 6 indicated that the pattern of similarity of species and functions of soil microbial communities along an altitudinal gradient in a P. massoniana forest was basically the same as described above. RDA ordination of soil microbial communities at different altitudes based on functional gene composition showed a good level of consistency, which was slightly different from the ordination based on NR species. The distribution of NR species, NOG, KO and CAZy functional genes of soil microbial community at MP1000, MP1400, and MP1600 were clearly separated from those observed at MP1200. Furthermore, a significant difference was observed between the $P$. massoniana forest and the tea garden surveyed.

\subsection{Co-occurrence Patterns of Different Soil Microbial Groups}

We detected significant differences among different soil microbial groups (archaea, bacteria, fungi, and viruses) and their corresponding random networks (Fig. 7) in terms of average degree, modularity, number of communities, and degree centrality (Table 4$)$. Modularity ranked as follows: archaea $(0.866)>$ bacteria (0.794) > fungi $(0.698)>$ viruses $(0.539)$, while degree centrality showed the following ranking: bacteria $(0.181)>$ viruses $(0.155)>$ fungi $(0.087)>$ archaea $(0.048)$ (Table 4). Overall, the co-occurrence network of archaeal and fungal species showed a relatively higher similarity, which may be related to the origin of the relationship between archaea and fungi. In turn, the co-occurrence network of bacterial and viral species showed a relatively higher similarity, which may be due to the closer relationship between them in the life history of the individuals. Overall, these data indicate a challenging and complex ecological network of relationships among different groups of soil microorganisms that warrants further in-depth study.

\section{Discussion}

The relationship between altitudinal distribution patterns and maintenance of biodiversity is currently a highly controversial topic in the field of biodiversity and ecosystem functions. The Leigong Mountain belongs to a typical mid-subtropical mountain forest ecosystem in southwestern China declared a National Nature Reserve, whose ecological environment is well preserved, thus providing an ideal site for the "an ideal site for the in-situ experiment" reported herein. In addition, previous studies at the site provided us with important and useful information on soil microorganism diversity and vegetation types along the elevation gradient of Leigong Mountain. Such information revealed great differences in soil microbiome and vegetation diversity at each elevation step. Therefore, we hypothesized that the variation in soil microorganism communities at different elevations may be affected by the variation in vegetation types.

P. massoniana is a native and widely distributed tree species that is well adapted to the regional climatic conditions; this ensured the selection of representative $P$. massoniana forest plots along the altitudinal 
gradient established for conducting the survey reported herein. At the same time, soil microbial communities can be diagnosed by metagenome technology, which can annotate microorganisms to the species level, and detect archaea, bacteria, fungi, and viruses in soil microbiomes; further, this technology can perform genetic function annotations, thereby providing richer biological information for the structural and functional characteristics of microbial communities among different elevations.

Euryarchaeota and Thaumarchaeota were the predominant phylum of soil archaea in the P. massoniana forest across different altitude gradients. A large number of reports have pointed that they are the dominant phylum of soil archaea, making important contributions to the biogeochemical cycles of soil carbon, nitrogen, and hydrogen, among many other elements. A good example of this paramount ecological function is the ammonia oxidation by autotrophic ammonia-oxidizing archaea as a key step in the nitrogen digestion process in soils (Leininger, et al. 2006; Zhang et al. 2012). In addition, this study found that the abundance of archaea increased with increasing altitude in the range of 1000-1600 m a.s.l., on Leigong Mountain. This finding is consistent with results reported for the range of 1000-1500 m a.s.l., on Mount Fuji, in Japan (Singh et al. 2012).

Bacteria are the largest and most diverse and versatile of soil microbial groups (Delgado-Baquerizo et al. 2018). Consistently with the general consensus that they are the most common phylum among soil bacteria (Wang et al. 2015), in this study Proteobacteria and Acidobacteria were the overwhelmingly predominant soil bacterial groups, both playing a very important role in the soil-matter cycle (carbon, nitrogen, sulphur, and other elements) and in the construction of the ecological environment (Barns et al. 2007, Spain et al. 2009).

Fungi are another important component of soil microbiome, as they play a crucial role in nutrient cycling, as well as in promoting plant growth and vegetation community succession. Consistently with previous reports (Matsuoka et al. 2019), in this study Ascomycota and Basidiomycota were the predominant fungal groups across the altitudinal gradient under study; however, they showed opposite trends in relative abundance along the altitude gradient; thus, the relative abundance of Ascomycota decreased with increasing elevation (from $66.9 \%$ at Mp1000 to $46.3 \%$ at Mp1600), whereas Basidiomycota increased with increasing elevation (from $25.2 \%$ at Mp1000 to $48.1 \%$ at Mp1600); still, the overall relative abundance of both Ascomycota and Basidiomycota together also increased slightly with elevation (92.2\% at Mp1000 to $94.4 \%$ at Mp1600). The increasing trend in relative abundance observed herein for Basidiomycota is consistent with the increasing trend observed for the same group in the altitude range of 950-1700 m a.s.l., in Norikura Mountain, in Japan (Ogwu et al. 2019). Furthermore, the change trend observed here for Ascomycota was also similar in both studies. Therefore, the specific set of predominant fungal groups may serve as a more sensitive indicator of environmental gradients determined by differences in altitude.

Viruses are ubiquitous and play important roles in regulating their hosts' mortality and community structure, the genetic landscape, and the nutrient turnover in ecosystem (Anderson et al. 2011; PaezEspino et al. 2016; Narr et al. 2017). Although soil is the most important habitat for virus distribution, 
there is currently a very limited number of reports on soil viruses (Paez-Espino et al. 2016; Narr et al. 2017), which may be due to the challenges involved in the detection, isolation, and classification of unknown viruses (Narr et al. 2017); therefore, viruses constitute what is referred to as, "the dark matter" of soil microbial communities (Paez-Espino et al. 2016). In this study, Myoviridae, Podoviridae and Siphoviridae, were the predominant virus groups in the soils of the $P$. massoniana forest of Leigong Mountain. Although they are reportedly the most common virus groups in a wide range of environments (Chen et al. 2014); we found no obvious trend in viral community-composition changes along between 1000 and $1600 \mathrm{~m}$ a.s.l., in our study. This result likely reflects the fact that virus dynamics may be affected by multiple factors, including host and environmental factors. The results of our symbiotic network analysis further intuitively reflect the differences in interaction patterns among species within different groups of the microbial community in a soil.

The gene functional composition of the soil microbial community at different altitudes was predicted by NOG, KEGG, and CAZ functional gene annotations. The results showed a relatively consistent trend among these different functional gene analyses. Thus, there were no obvious differences at the high category level in functional gene detection along the elevation gradient surveyed, a finding that may be related to the phenomenon of "gene redundancy" (Fierer et al. 2013); which consists in soil microbial communities containing similar functional genes despite even significant differences in community species composition in response to environmental changes. Nonetheless, our comparison between species composition and functional gene composition matrix of the soil microbial communities along the altitudinal gradient surveyed showed a significant correlation between the two, which supports our hypothesis that soil microbiome diversity can influence multiple ecosystem functions, while the elevation factor may indirectly influence soil microbiome functions by shaping soil microbiome composition.

\section{Conclusions}

Our findings suggest that different types of soil microorganisms over the altitudinal gradient have different distribution patterns in a P. massoniana forest. However, Leigong Mountain is a typical midsubtropical mountain forest ecosystem that shows an overall altitude gradient from 650-2179 $\mathrm{m}$ a.s.l., within which our study focused the range from 1000 to $1600 \mathrm{~m}$ a.s.l. In the future, studies on the pattern of soil microbial distribution should disentangle the inner links between the environmental variables (vegetation types, edaphic factor, microclimate, etc.) and the detailed functions of the microbial communities along wider elevation gradients in this typical sub-tropical, non-karst mountain subtropical forest ecosystem.

\section{Abbreviations}

NOG: nonsupervised orthologous groups; KEGG: the Kyoto encyclopedia of genes and genomes-based pathways; SOC: soil organic carbon; TN: total nitrogen; TP: total phosphorus; DNA: deoxyribonucleic acid; ORF: open reading frames; NR: non-redundant protein sequences; CAZy: carbohydrate-active enzymes; FUNGuild囚an open annotation tool for parsing fungal community datasets by ecological guild; RDA囚 
redundancy analysis; GT: glycosyl transferase; GH: glycoside hydrolase; CE: carbohydrate esterase; AA: coenzyme activity; PL: polysaccharide lyase; CBM: carbohydrate module

\section{Declarations}

\section{Acknowledgments}

We wish to thank the editor and two anonymous reviewers for their constructive comments and suggestions for improving this manuscript.

\section{Authors' contributions}

Jian Zhang, Ming Xu and Xiao Zou conceived the study; Jian Zhang performed the analyses and wrote the first draft; all authors contributed critically to successive drafts and gave final approval for publication.

\section{Funding}

This study was supported by the National Natural Science Foundation of China (31660150, 31960234, 31860037), the Natural Science Foundation of Guizhou Province, China (Qian Ke He Basic Project [2017]1059, Qian Ke He Supporting Project [2018]2773, Qian Ke He Major Project [2019]3005-4), the Construction Program of Biology First-class Discipline in Guizhou (GNYL [2017]009).

\section{Availability of data and materials}

The datasets used and/or analyzed in during the current study are available from the corresponding author on reasonable request.

\section{Ethics approval and consent to participate}

No applicable.

\section{Consent for publication}

No applicable.

\section{Competing interests}

The authors declare that they have no competing interests.

\section{References}

Altschul SF, Madden TL, Schaffer AA, Zhang J, Zhang Z, Miller W, Lipman DJ (1997): Gapped BLAST and PSI-BLAST: a new generation of protein database search programs. Nucleic Acids Research 25, 3389-402 
Anderson RE, Brazelton WJ, Baross JA (2011): Is the genetic landscape of the deep subsurface biosphere affected by viruses? Frontiers in Microbiology 2

Bahram M et al. (2018): Structure and function of the global topsoil microbiome. Nature 560, 233-+

Barberan A, Bates ST, Casamayor EO, Fierer N (2012): Using network analysis to explore co-occurrence patterns in soil microbial communities. ISME Journal 6, 343-351

Bardgett RD, van der Putten WH (2014): Belowground biodiversity and ecosystem functioning. Nature $515,505-511$

Barns SM, Cain EC, Sommerville L, Kuske CR (2007): Acidobacteria phylum sequences in uraniumcontaminated subsurface sediments greatly expand the known diversity within the phylum. Applied and environmental microbiology 73, 3113-3116

Chen L, Xun W, Sun L, Zhang N, Shen Q, Zhang R (2014): Effect of different long-term fertilization regimes on the viral community in an agricultural soil of Southern China. European Journal of Soil Biology 62, $121-126$

Delgado-Baquerizo M, Oliverio AM, Brewer TE, Benavent-González A, Eldridge DJ, Bardgett RD, Maestre FT, Singh BK, Fierer N (2018): A global atlas of the dominant bacteria found in soil. Science 359, 320-325

Du J, Yuan Z, Ma Z, Song J, Xie X, Chen Y (2014): KEGG-PATH: Kyoto encyclopedia of genes and genomes-based pathway analysis using a path analysis model. Molecular Biosystems 10, 2441-2447

Fierer N, Ladau J, Clemente JC, Leff JW, Owens SM, Pollard KS, Knight R, Gilbert JA, McCulley RL (2013): Reconstructing the Microbial Diversity and Function of Pre-Agricultural Tallgrass Prairie Soils in the United States. Science 342, 621-624

Geml J, Morgado LN, Semenova-Nelsen TA, Schilthuizen M (2017): Changes in richness and community composition of ectomycorrhizal fungi among altitudinal vegetation types on Mount Kinabalu in Borneo. New Phytologist 215, 454-468

Jarvis SG, Woodward S, Taylor AFS (2015): Strong altitudinal partitioning in the distributions of ectomycorrhizal fungi along a short (300 m) elevation gradient. New Phytologist 206, 1145-1155

Körner C (2007): The use of 'altitude' in ecological research. Trends in Ecology \& Evolution 22, 569-574

Lei L, Xiao W, Zeng L, Zhu J, Huang Z, Cheng R, Gao S, Li M-H (2018): Thinning but not understory removal increased heterotrophic respiration and total soil respiration in Pinus massoniana stands. The Science of the total environment $621,1360-1369$

Leininger S, Urich T, Schloter M, Schwark L, Qi J, Nicol GW, Prosser JI, Schuster SC, Schleper C (2006): Archaea predominate among ammonia-oxidizing prokaryotes in soils. Nature 442, 806-809 
Li D, Liu C-M, Luo R, Sadakane K, Lam T-W (2015): MEGAHIT: an ultra-fast single-node solution for large and complex metagenomics assembly via succinct de Bruijn graph. Bioinformatics 31, 1674-1676

Liu D, Liu G, Chen L, Wang J, Zhang L (2018): Soil pH determines fungal diversity along an elevation gradient in Southwestern China. Science China Life Sciences 61, 718-726

Lladó S, López-Mondéjar R, Baldrian P (2018): Drivers of microbial community structure in forest soils. Applied Microbiology and Biotechnology 102, 4331-4338

Lombard V, Ramulu HG, Drula E, Coutinho PM, Henrissat B (2014): The carbohydrate-active enzymes database (CAZy) in 2013. Nucleic Acids Research 42, D490-D495

Luo R et al. (2012): SOAPdenovo2: an empirically improved memory-efficient short-read de novo assembler. Gigascience 1

Matsuoka S, Ogisu Y, Sakoh S, Hobara S, Osono T (2019): Taxonomic, functional, and phylogenetic diversity of fungi along primary successional and elevational gradients near Mount Robson, British Columbia. Polar Science 21, 165-171

Myers N, Mittermeier RA, Mittermeier CG, da Fonseca GAB, Kent J (2000): Biodiversity hotspots for conservation priorities. Nature 403, 853-858

Narr A, Nawaz A, Wick LY, Harms H, Chatzinotas A (2017): Soil Viral Communities Vary Temporally and along a Land Use Transect as Revealed by Virus-Like Particle Counting and a Modified Community Fingerprinting Approach (fRAPD). Frontiers in Microbiology 8

Noguchi H, Park J, Takagi T (2006): MetaGene: prokaryotic gene finding from environmental genome shotgun sequences. Nucleic Acids Research 34, 5623-30

Ogwu MC, Takahashi K, Dong K, Song H-K, Moroenyane I, Waldman B, Adams JM (2019): Fungal Elevational Rapoport pattern from a High Mountain in Japan. Scientific Reports 9

Pabinger S, Dander A, Fischer M, Snajder R, Sperk M, Efremova M, Krabichler B, Speicher MR, Zschocke J, Trajanoski $Z$ (2014): A survey of tools for variant analysis of next-generation genome sequencing data. Briefings in Bioinformatics 15, 256-278

Paez-Espino D, Eloe-Fadrosh EA, Pavlopoulos GA, Thomas AD, Huntemann M, Mikhailova N, Rubin E, Ivanova NN, Kyrpides NC (2016): Uncovering Earth's virome. Nature 536, 425-430

Powell S, Szklarczyk D, Trachana K, Roth A, Kuhn M, Muller J, Arnold R, Rattei T, Letunic I, Doerks T, Jensen LJ, von Mering C, Bork P (2012): eggNOG v3.0: orthologous groups covering 1133 organisms at 41 different taxonomic ranges. Nucleic Acids Research 40, D284-D289 
Qiu B, Zhong M, Zeng C, Tang Z, Chen C (2012): Effect of topography and accessibility on vegetation dynamic pattern in Mountain-hill Region. Journal of Mountain Science 9, 879-890

Schirmer M, ljaz UZ, D'Amore R, Hall N, Sloan WT, Quince C (2015): Insight into biases and sequencing errors for amplicon sequencing with the Illumina MiSeq platform. Nucleic Acids Research 43

Schloter M, Nannipieri P, Sorensen SJ, van Elsas JD (2018): Microbial indicators for soil quality. Biol. Fertil. Soils 54, 1-10

Schoen ME, Nieselt K, Garnica S (2018): Belowground fungal community diversity and composition associated with Norway spruce along an altitudinal gradient. Plos One 13

Siles JA, Margesin R (2016): Abundance and diversity of bacterial, archaeal, and fungal communities along an altitudinal gradient in alpine forest soils: What are the driving factors? Microbial Ecology 72 , 207-220

Singh D, Takahashi K, Adams J (2012): Elevational Patterns in Archaeal Diversity on Mt. Fuji. PloS one 7, e44494

Spain AM, Krumholz LR, Elshahed MS (2009): Abundance, composition, diversity and novelty of soil Proteobacteria. ISME Journal 3, 992-1000

Tajik S, Ayoubi S, Lorenz N (2020): Soil microbial communities affected by vegetation, topography and soil properties in a forest ecosystem. Applied Soil Ecology 149

Thakur MP, Geisen S (2019): Trophic Regulations of the Soil Microbiome. Trends Microbiol. 27, 771-780 van der Heijden MGA, Wagg C (2013): Soil microbial diversity and agro-ecosystem functioning. Plant and Soil $363,1-5$

Wang J-T, Cao P, Hu H-W, Li J, Han L-L, Zhang L-M, Zheng Y-M, He J-Z (2015): Altitudinal Distribution Patterns of Soil Bacterial and Archaeal Communities Along Mt. Shegyla on the Tibetan Plateau. Microbial Ecology 69, 135-145

Xu M, Zhang J, Liu GB, Yamanaka N (2014): Soil properties in natural grassland, Caragana korshinskii planted shrubland, and Robinia pseudoacacia planted forest in gullies on the hilly Loess Plateau, China. Catena 119, 116-124

Zhang H, Duan F, Li Y, Wang Q, Lu X, Gan X, Xie Z, Tang J (2020): Population structure and quantitative characteristics of Tetracentron sinense (Trochodendraceae) in Leigong mountain nature reserve, China. Botanical Sciences 98, 86-100

Zhang J, Taniguchi T, Tateno R, Xu M, Du S, Liu GB, Yamanaka N (2013): Ectomycorrhizal fungal communities of Quercus liaotungensis along local slopes in the temperate oak forests on the Loess 
Plateau, China. Ecological Research 28, 297-305

Zhang L-M, Hu H-W, Shen J-P, He J-Z (2012): Ammonia-oxidizing archaea have more important role than ammonia-oxidizing bacteria in ammonia oxidation of strongly acidic soils. The ISME Journal $6,1032-$ 1045

\section{Tables}

Table 1 Basic information of study sites

\begin{tabular}{|c|c|c|c|c|c|}
\hline Sites & Latitude and longitude & $\begin{array}{l}\text { Altitude } \\
\bigotimes \mathrm{m} \rrbracket\end{array}$ & $\begin{array}{l}\text { Slope } \\
\nabla^{\circ} \rrbracket\end{array}$ & $\begin{array}{l}\text { Aspect } \\
\nabla^{\circ} \mathbb{\square}\end{array}$ & Dominant species \\
\hline Mp1000 & $\begin{array}{l}108^{\circ} 06^{\prime} 42.08^{\prime \prime} \\
26^{\circ} 23^{\prime} 00.72^{\prime \prime}\end{array}$ & 1008 & 12 & 121 & $\begin{array}{l}\text { Pinus } \\
\text { Massoniana+Cunninghamia } \\
\text { Lanceolata }\end{array}$ \\
\hline Mp1200 & $\begin{array}{l}108^{\circ} 10^{\prime} 02.83^{\prime \prime} \\
26^{\circ} 21^{\prime} 29.72^{\prime \prime}\end{array}$ & 1198 & 10 & 160 & P. massoniana \\
\hline Mp1400 & $\begin{array}{l}108^{\circ} 10^{\prime} 04.86^{\prime \prime} \\
26^{\circ} 21^{\prime} 40.68^{\prime \prime}\end{array}$ & 1394 & 15 & 141 & P. massoniana \\
\hline Mp1600 & $\begin{array}{l}108^{\circ} 11^{\prime} 30.85^{\prime \prime} \\
26^{\circ} 22^{\prime} 27.92^{\prime \prime}\end{array}$ & 1596 & 7 & 165 & P. massoniana+C. Lanceolata \\
\hline $\begin{array}{l}\text { Tea- } \\
\text { garden }\end{array}$ & $\begin{array}{l}108^{\circ} 06^{\prime} 42.21^{\prime \prime}, \\
26^{\circ} 23^{\prime} 01.73^{\prime \prime}\end{array}$ & 991 & 3 & 120 & Camellia sinensis \\
\hline
\end{tabular}

Table 2 Statistics of metagenomic sequencing by NR species annotation

\begin{tabular}{|lllllll|}
\hline Microbe & Phylum & Class & Order & Family & Genus & Species \\
\hline Aracheae & 12 & 24 & 38 & 60 & 144 & 912 \\
\hline Bacteria & 82 & 135 & 251 & 466 & 1864 & 11576 \\
\hline Fungi & 10 & 33 & 74 & 154 & 254 & 417 \\
\hline Viruses & 1 & 1 & 4 & 17 & 60 & 402 \\
\hline Total & 105 & 193 & 367 & 697 & 2322 & 13307 \\
\hline
\end{tabular}

Table 3 Alpha diversity of soil microbial community 


\begin{tabular}{|llllllllll|}
\hline Sites & \multicolumn{2}{l}{ Richness } & \multicolumn{7}{c|}{ Shannon index } \\
\cline { 2 - 9 } & Archaea & Bacteria & Fungi & Viruses & Archaea & Bacteria & Fungi & Viruses \\
\hline Mp1000 & 689 & 9993 & 292 & 125 & 7.29 & 9.08 & 7.3 & 4.72 \\
\hline Mp1200 & 752 & 10172 & 297 & 166 & 7.64 & 8.62 & 7.16 & 4.45 \\
\hline Mp1400 & 698 & 10247 & 318 & 138 & 7.85 & 8.97 & 7.29 & 4.91 \\
\hline Mp1600 & 716 & 10114 & 297 & 118 & 7.86 & 8.9 & 7.12 & 4.06 \\
\hline Tea-garden & 697 & 10348 & 354 & 225 & 7.03 & 8.45 & 7.51 & 5.7 \\
\hline
\end{tabular}

Table 4 Topological properties of soil microbiome co-occurrence network

\begin{tabular}{|lllll|}
\hline Network properties & Archaea & Bacteria & Fungi & Viruses \\
\hline Number of nodes & 527 & 748 & 399 & 236 \\
\hline Positive edges & 2589 & 12308 & 1762 & 6952 \\
\hline Negative edges & 494 & 663 & 144 & 15 \\
\hline Average degree & 11.7 & 34.7 & 9.6 & 37.1 \\
\hline Modularity & 0.866 & 0.794 & 0.698 & 0.539 \\
\hline Number of communities & 23 & 53 & 38 & 34 \\
\hline Degree centralization & 0.048 & 0.181 & 0.087 & 0.155 \\
\hline
\end{tabular}

\section{Figures}

(a) Archaea

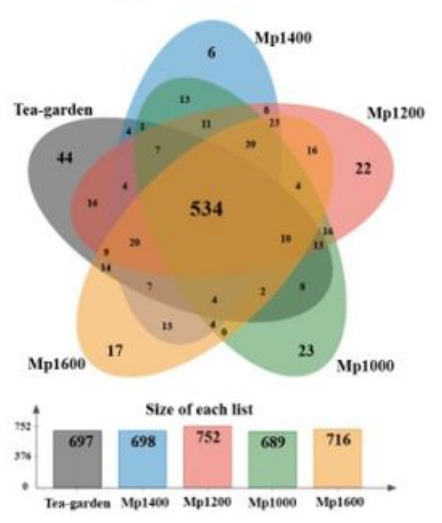

(b) Bacteria

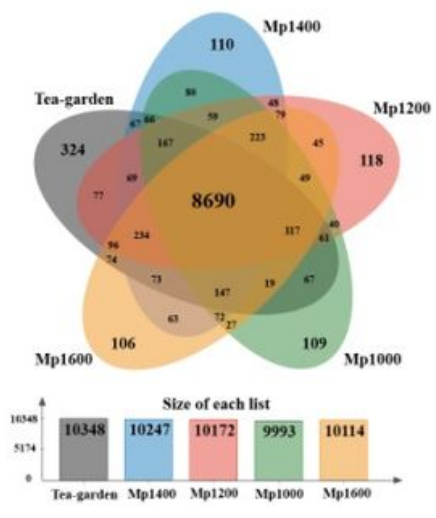

(c) Fungi

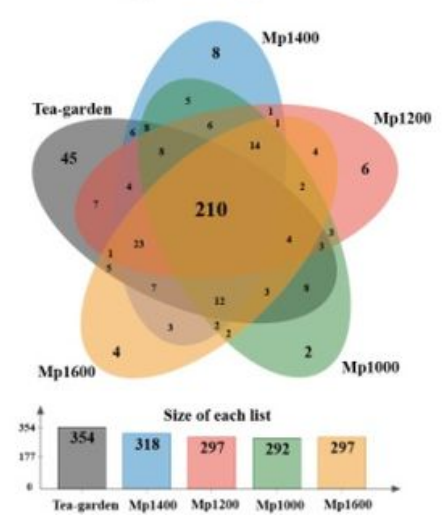

(d) Viruses

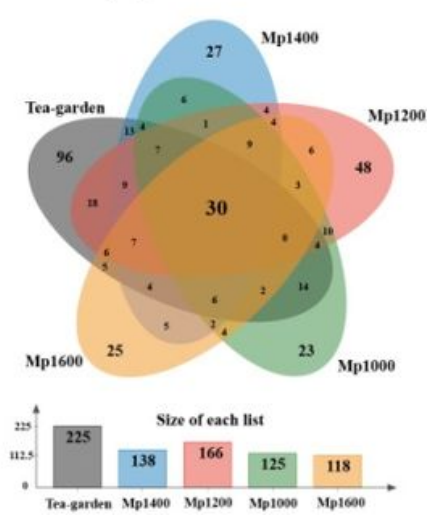

Figure 1 
Species composition of archaea, bacteria, fungi, and viruses in soil microbial communities at different elevations in a Pinus massoniana forest.

(a) Archaea

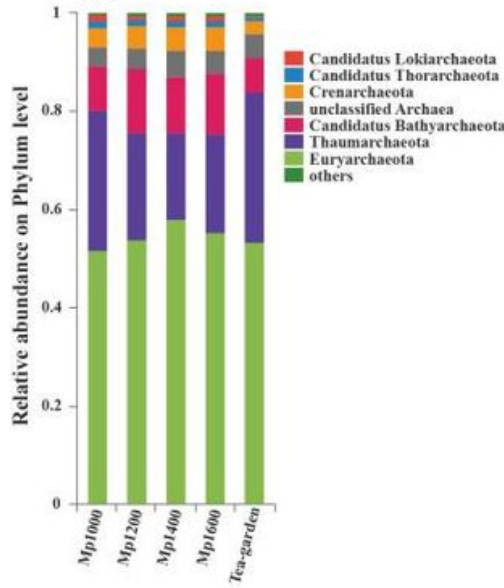

(b) Bacteria

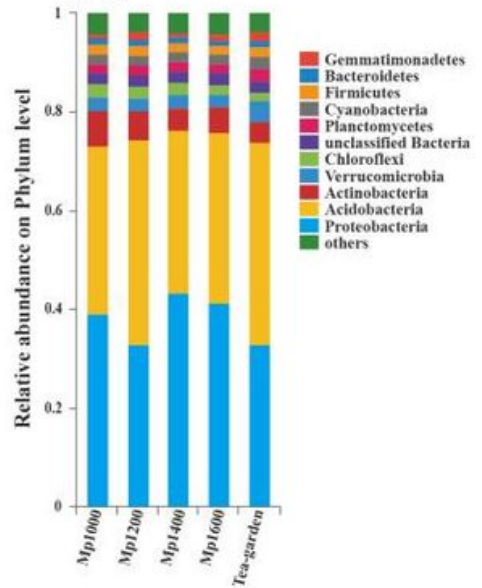

(c) Fungi

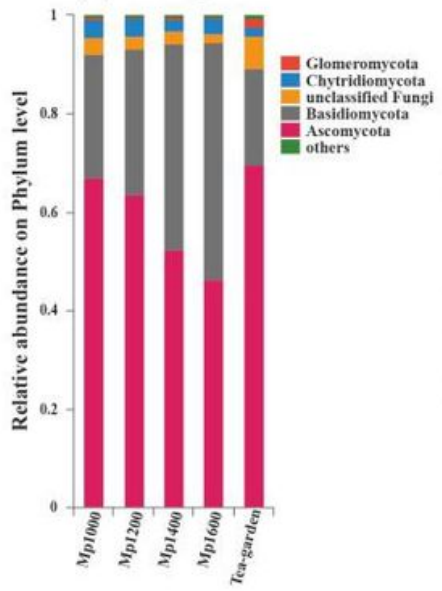

(d) Viruses

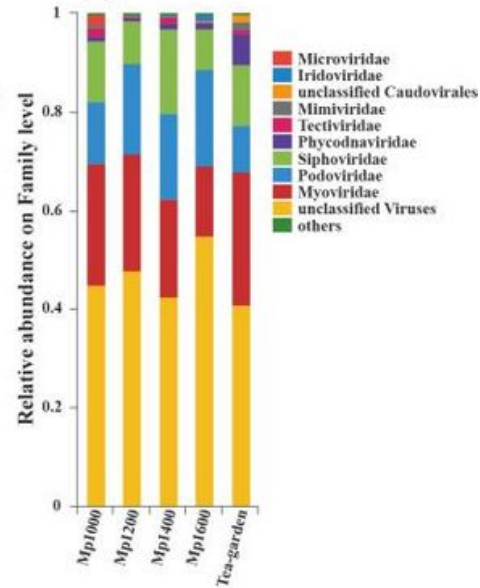

Figure 2

The relative abundance of (a)Archaea, (b) Bacteria, (c) Fungi, (d) Viruses in soil microbial communities across an altitudinal gradient in a Pinus massoniana forest.

(a) Archaea

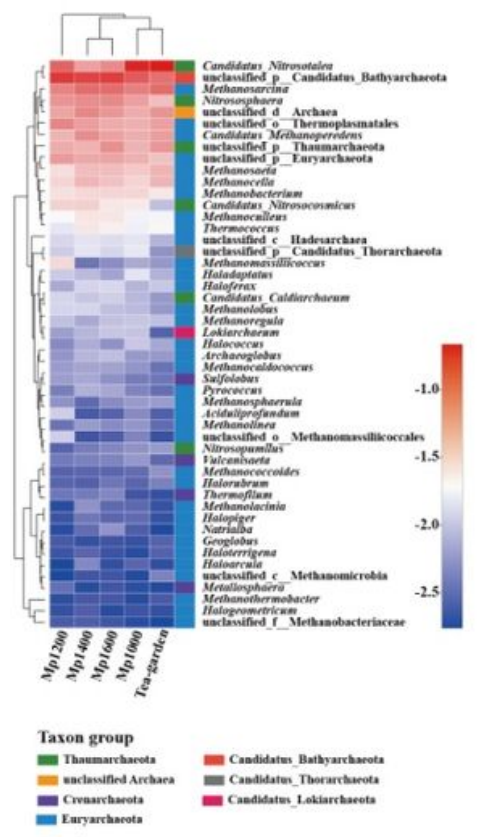

(b) Bacteria

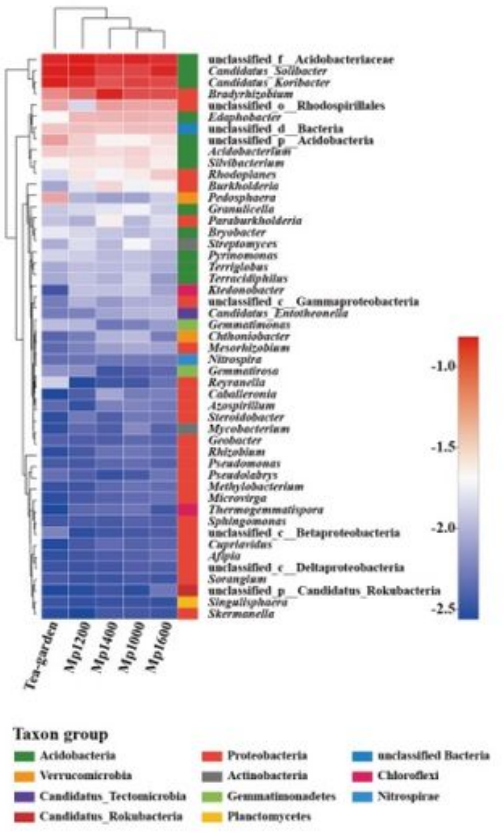

(c) Fungi

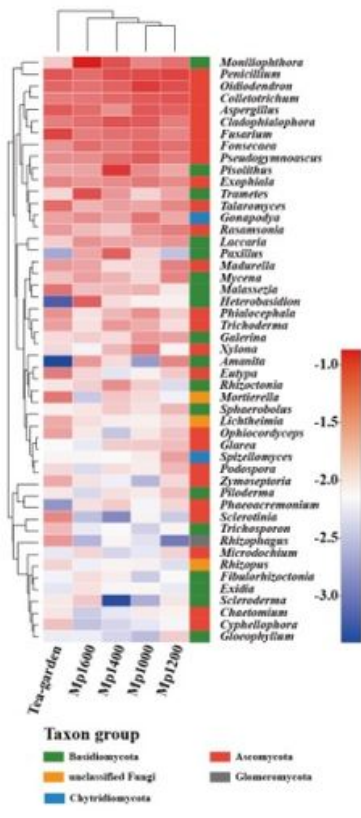

(d) Viruses

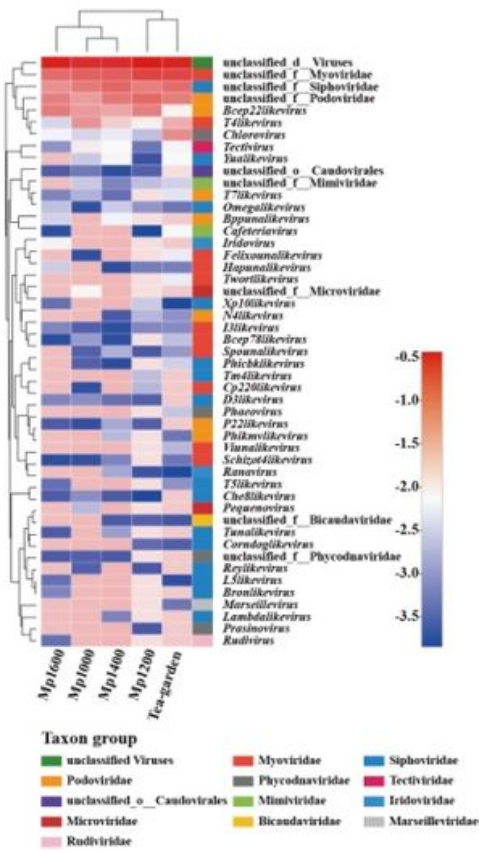

\section{Figure 3}

Relationship between (a) Archaea, (b) Bacteria, (c) Fungi, and (d) Viruses genera present in forest-soil microbial communities, and altitude. 


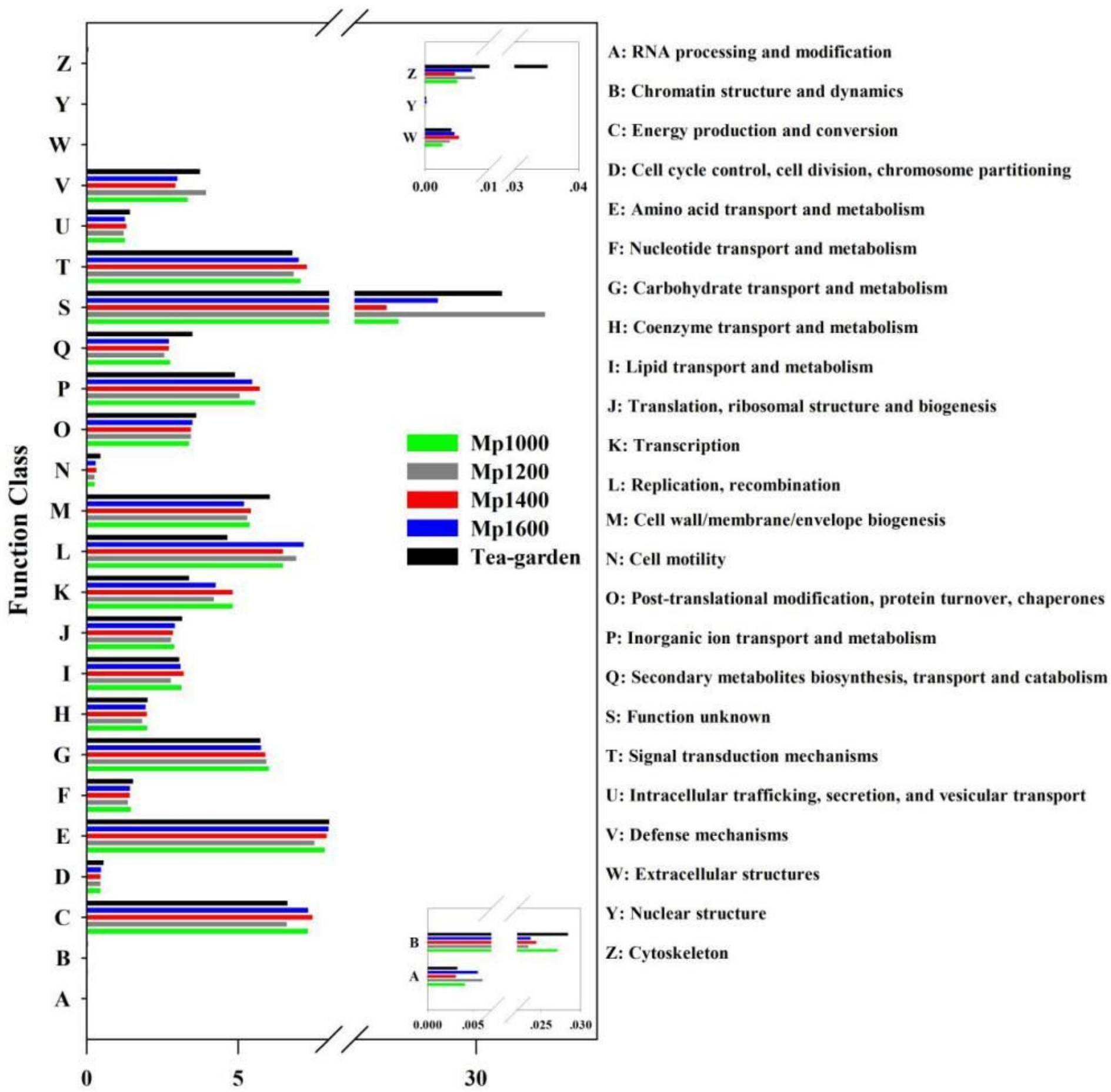

Relative abundance (\%)

\section{Figure 4}

NOG functional gene composition of soil microbial communities at different elevations in a Pinus massoniana forest. 

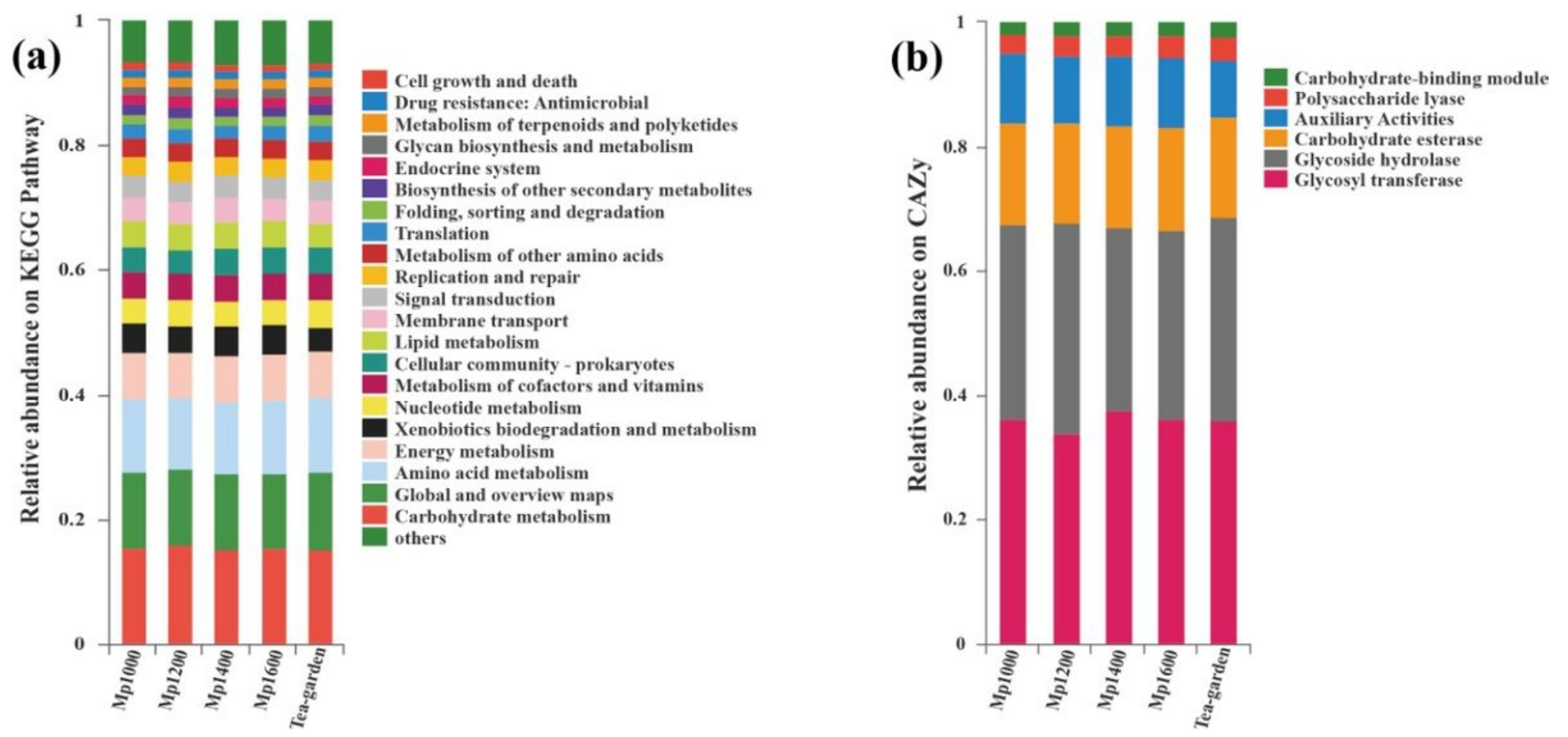

Figure 5

KEGG-based CAZy functional gene classification for soil microbial communities at different elevations in a Pinus massoniana forest.

(a) NR物种

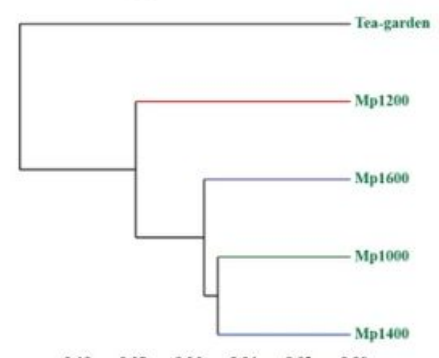

$\begin{array}{llllll}0.10 & 0.08 & 0.06 & 0.04 & 0.02 & 0.00\end{array}$

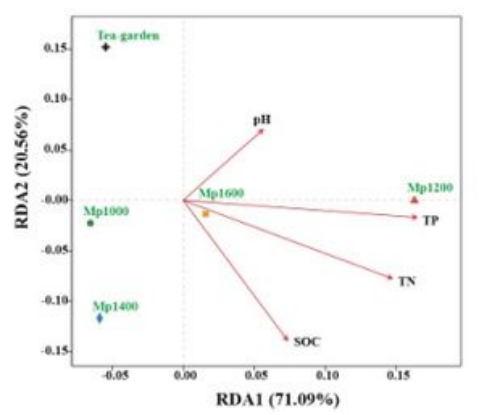

(b) NOG 功能
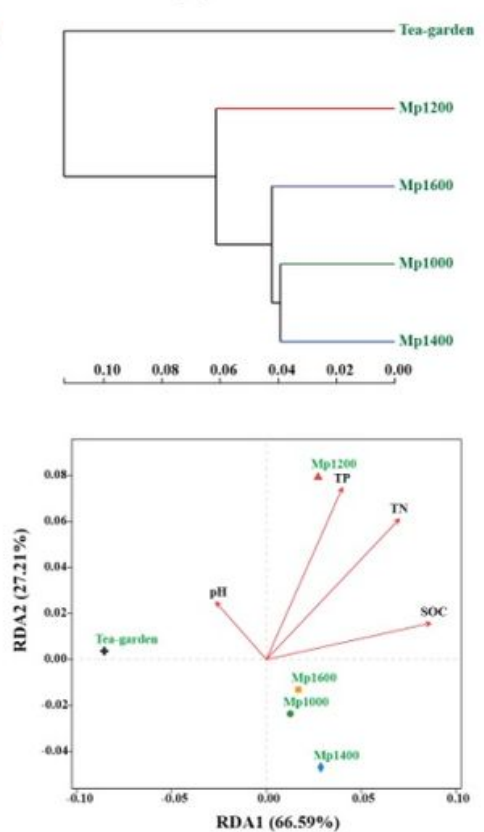

(d) KO功能
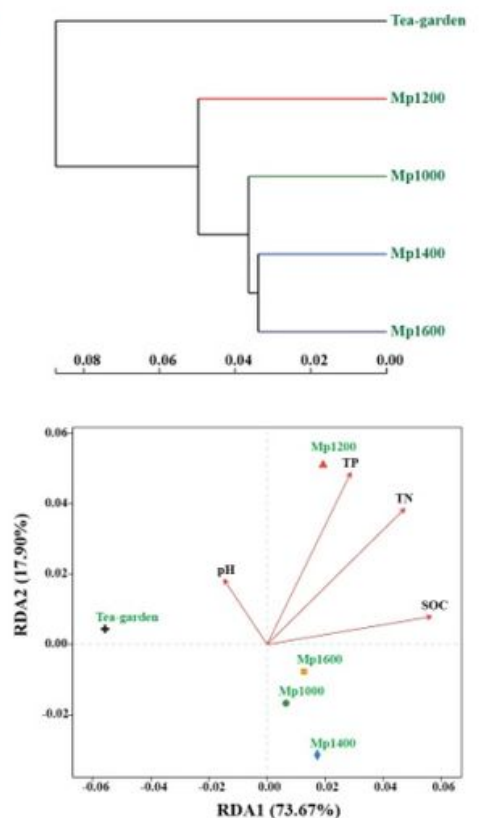

(d) CAZy 功能
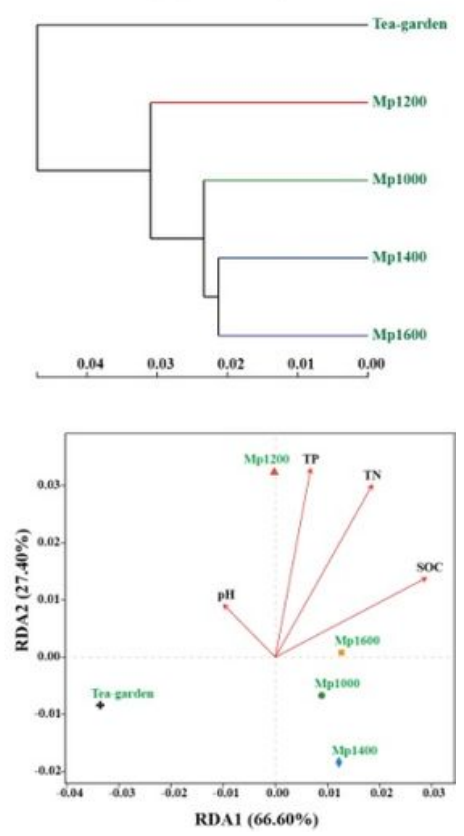

Figure 6

(a) NR species, (b) NOG, (c) KO, and (d) CAZy classification of functional genes of soil microbial communities by clustering tree and RDA analyses 

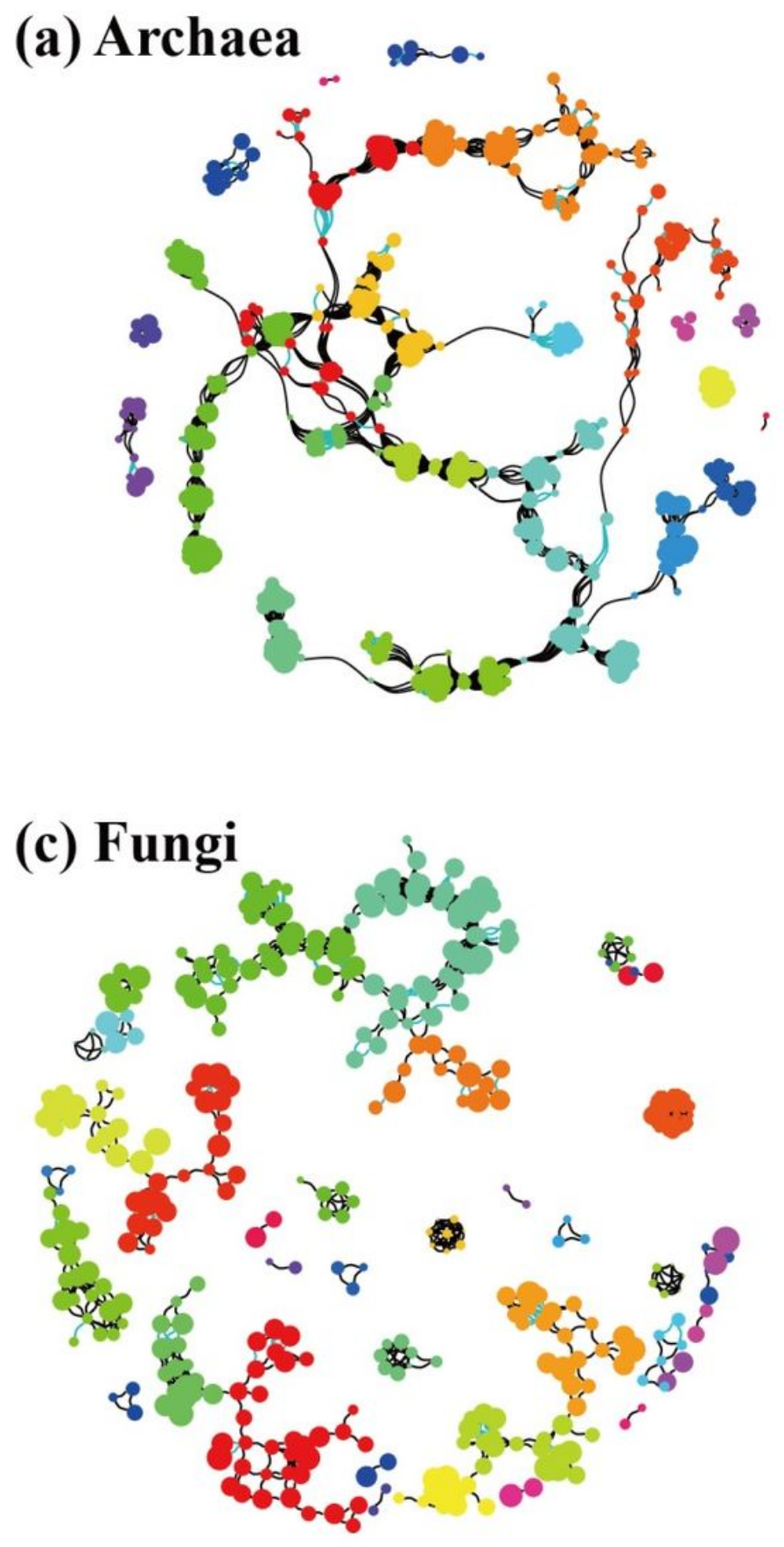

(b) Bacteria

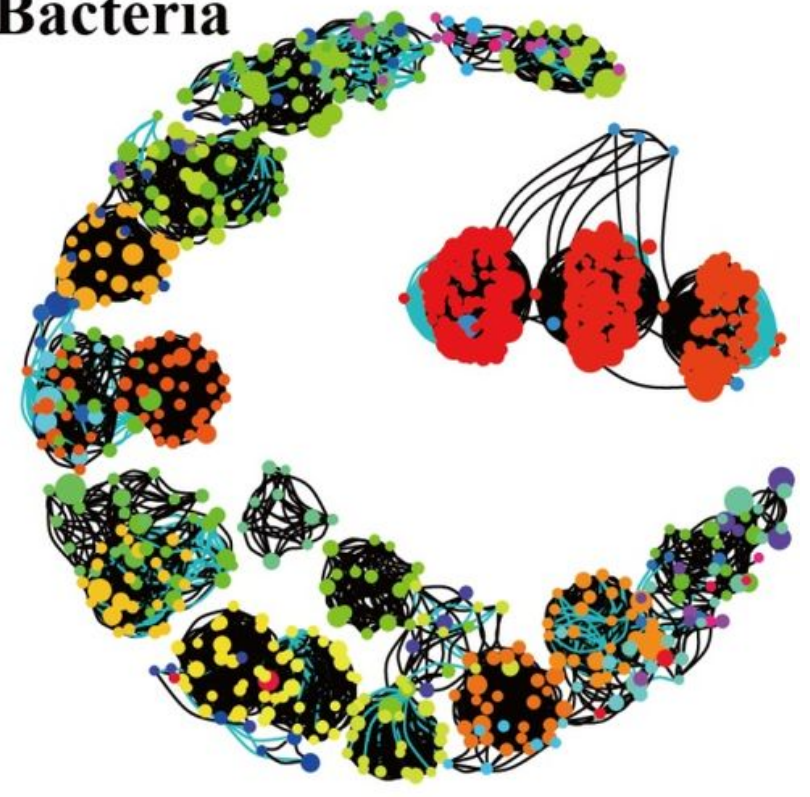

(d) Viruses

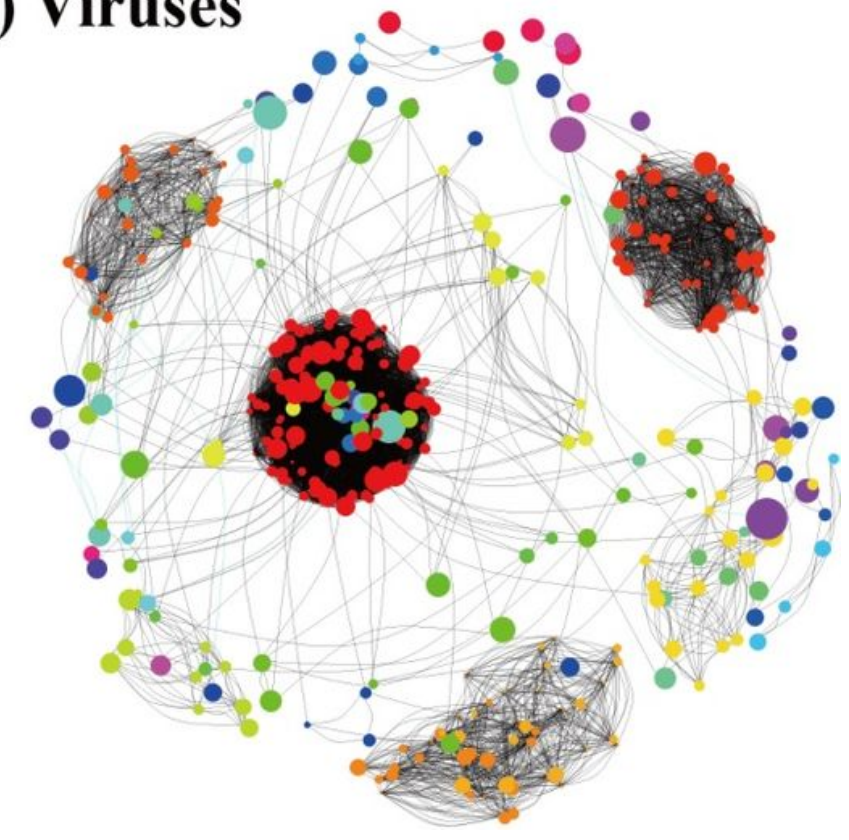

Figure 7

Species co-occurrence networks of different groups of soil microorganisms (a. archaea; b. bacteria; c. fungi; d. viruses) 